\title{
Estados de ánimo precompetitivos y resultados deportivos en jugadores de bolos cántabros
}

\section{Mood states of pre-competitive and sports results in cantabrian bowling players}

\author{
Estados de humor de pré-competitivo e resultados \\ desportivos em jogadores do boliche cantábrico
}

\author{
J. Díaz', J.N. Gutiérrez² y J.A. Hoyos ${ }^{3}$ \\ ${ }^{1}$ Asociación Cántabra de Psicología del Deporte. APD-Cantabria, ${ }^{2}$ Universidad de Cantabria. Departamento de Educación y ${ }^{3}$ Dirección Técnica de la \\ Federación Cántabra de Bolos-FCB.
}

Resumen: En este trabajo se analizan y describen los estados de ánimo precompetitivos de los jugadores de la especialidad de bolo-palma obtenidos con las pruebas de Perfil de Estados de Ánimo Profile of Mood States (POMS). La muestra la componen los 13 jugadores de bolos de la primera categoría de Cantabria que jugaron la fase final del LXXIV Campeonato Regional de Bolos. Se han realizado los gráficos de los perfiles de los estados de ánimos precompetitivos y se han analizado según los resultados y clasificación conseguida. Se ha elaborado un estudio correlacional entre los factores del POMS, así como contrastes en función de t de Student y se han comentado las diferencias entre los jugadores según la edad, experiencia competitiva y haber jugado o no un concurso previo minutos antes de cumplimentar el cuestionario. Los resultados muestran que el perfil iceberg es el predominante en los jugadores que jugaron la fase final del campeonato. La mejor clasificación y resultados la consiguen los jugadores con perfiles de estado de ánimo precompetitivos más idóneos que son también los jugadores con mayor experiencia competitiva y más edad.

Palabras clave: Análisis, Estados de ánimo, Precompetición, Bolos, Resultados

Abstract: In this paper, bolo-palma player's pre-competition mood is analyzed and described. These different moods are obtained through the test of the Profile of Mood States (POMS). The sample is made up of 13 bolos players within the highest division in Cantabria that took part in the final stage of the $74^{\text {th }}$ Regional Boliche Cantábrico Championship. The graphic of the profiles of the pre-competition moods obtained have been carried out and analyzed based on the results and the final classification of the players. A correlational study between the factors of POMS has been developed, as well as the contrast based on the $\mathrm{t}$ Student and the differences between players have been analyzed considering the age, the competition experience and the fact of having played right before filling out the questionnaire. The results show that the so called iceberg profile is predominant in players who took part in the final stage of the championship. The best classification and results are obtained by players whose pre-competition mood is more suitable and these player tend to be those with longer experience and older Key words: analysis, mood, pre-competition, bolos, results.

Resumo: Neste trabalho, analisam-se e descrevem-se os estados de humor pré-competitivo dos jogadores da especialidade de bolo-palma obtidos com testes de Perfil de Estados de Humor Profile of Mood States (POMS). A amostra é composta pelos 13 jogadores da primeira categoria da Cantabria que jogaram a fase final do LXXIV Campeonato Regional de Bolos. Realizaram-se os gráficos dos perfis de estados de humor pré-competitivo e analisaram-se os resultados de acordo com a classificação conseguida. Estudaram-se as correlações entre os fatores do POMS, bem como os contrastes em função do teste $t$ de Student e comentaram-se as diferenças entre os jogadores de acordo com a idade, a experiência competitiva e ter ou não jogado um concurso prévio cinco minutos antes de responder ao questionário. Os resultados mostram que o Perfil Iceberg é predominante nos jogadores que jogaram a fase final do campeonato. A melhor classificação e os melhores resultados são conseguidos pelos jogadores com perfis de estados de humor pré-competitivo mais idóneos que são também os jogadores com mais experiência competitiva e mais idade.

Palavras-chave: Análise, estados de humor, pré-competição, bolos, resultados,

\section{Introducción}

Las percepciones que los deportistas tienen sobre sus estados de ánimo previos a las competiciones pueden influir, de alguna manera, sobre los diferentes aspectos de su rendimiento. Son numerosos los trabajos que, desde el ámbito de la psicología del deporte, han obtenido relaciones relevantes entre percepción de los estados de ánimo precompetitivos y ren-

Dirección para correspondencia [Correspondence address]: Joaquín Díaz Rodríguez. Asociación Cántabra de Psicología del Deporte APDCantabria. C/ Los Mártires, 13 6º Izda. 39300 Torrelavega. Cantabria (España).E-mail: quinichi@hotmail.com dimiento. (Arruza, Balagué y Arrieta, 1998; Lazarus, 2000; Ruiz y Hanin, 2004: Andrade, Arce y Seoane, 2000; De la Vega, Ruiz, García-Mas, Olmedilla y Del Valle, 2008; De la Vega, Galán, Ruiz y Tejero, 2013)

El cuestionario del Perfil de los Estados de Ánimo (POMS) en sus distintas adaptaciones al español -en este trabajo utilizamos la adaptación realizada por Fuentes, Balague, Meliá y García-Merita, (1995) de 29 ítems- es uno de los instrumentos más empleados en Psicología del Deporte (Leunes y Hayward, 1989) para identificar los niveles de los factores 
psicológicos, concretamente la percepción de los estados de ánimo precompetitivos, y su influencia en el rendimiento de los deportistas. Desde la aplicación del POMS en el deporte en el año 1975 ha demostrado ser el mejor predictor del éxito deportivo (Morgan 1980). Con este cuestionario se han realizado numerosos estudios, publicaciones y artículos, tanto en el ámbito del deporte de competición, como del ejercicio y en diferentes poblaciones y deportistas. El POMS, en sus distintas adaptaciones ha sido utilizado con profusión en muchas y variadas modalidades deportivas: Morgan, Costill, Flynn, Ranglin y O'Connor (1988). Nagle, Morgan, Hellickson, Serfass y Alexander (1975) en luchadores de élite. Morgan y Johson, (1977) en equipos de remo. Morgan y Pollok (1977) en corredores de fondo. Pérez, Solanas y Ferrer (1993) en nadadores de alto rendimiento. De la Vega, Ruiz, García y del Valle (2011) en fútbol profesional. De la Vega, Galán, Ruíz, y Tejero (2013) en Boccia Paralímpica. Sánchez, González, Ruíz, San Juan, Abando, De Nicolas et al. (2001) en jugadores de futbol. Segura, Molist, Arcarons, y Piqué (1999) en deportistas discapacitados físicos. Bonete, Moya y Suay (2009) con corredores de fondo y semifondo. Hernández, Torres-Luque y Olmedilla (2009) con judocas. Hassmen y Blomstrand (1995) en equipos de futbol. Terry (1995) en competiciones de Karate, Hockey y Boosling

En general, estos estudios han demostrado que en los deportistas de alto rendimiento los estados de ánimo previos a la competición y durante la competición muestran valores superiores en la escala de vigor, e inferiores en las de tensión, cólera, fatiga y depresión. Esta combinación de puntuación en estos factores dibuja una forma de perfil que Morgan (1980) denominó "perfil iceberg". Según este investigador ese tipo de perfil refleja un estado anímico idóneo para conseguir adecuados rendimientos. Por el contrario, aquellos deportistas que presentaran bajas puntuaciones en el factor vigor y fueran superiores las de los otros factores de tensión, cólera, fatiga o depresión, conseguirían peores rendimientos en las competiciones. (Andrade, Arce, y Simeone, 2000; Beedie, Terry y Lane, 2000).

El empleo del POMS no ha sido nunca utilizado en la modalidad del bolo-palma. (En lo sucesivo bolos) Con este trabajo incorporamos una modalidad deportiva nueva a los trabajos sobre el estado de ánimo percibido previo a la competición, y el rendimiento y resultado obtenido mediante el POMS

Los bolos es un deporte mentalmente muy exigente, muy complejo e implacable con los pequeños errores. Pocos deportes tienen una estructura competitiva que presente tanto atractivo para el trabajo mental. (Díaz, 2010) Los jugadores deben poseer una adecuada actitud mental (González, 2007) durante todo el tiempo que dura la competición. Existe un acuerdo general entre jugadores y expertos en bolos sobre la influencia que tienen los estados de ánimo previos a la competición para conseguir buenos rendimientos y registros.

Por lo que respecta al objetivo central de nuestro trabajo, el análisis y la descripción del estado de ánimo que presentaban los jugadores finalistas en el Campeonato Regional de bolos inmediatamente antes de comenzar a competir y la relación con los resultados, deberemos deliberar lo que representa el Campeonato Regional de bolos y la extraordinaria importancia que tiene para los jugadores.

El Campeonato Regional, en cuya fase final participan los mejores jugadores del Circuito de Bolos, es la suma de esfuerzos de toda la temporada. Está formado por 20 concursos puntuables y clasifican los 16 mejores participantes de estos 20 concursos. Cada concurso consta de un tiempo de 50 minutos aproximadamente. La fase final, obliga a los jugadores a permanecer prácticamente cinco horas en la bolera. Cuando no están jugando tienen que esperar la participación de sus rivales para saber si pasa el corte y continúa jugando o resulta eliminado. Las competiciones se tiran desde mayo a septiembre. La clasificación del Circuito de Bolos es, además, valedera para el mantenimiento de la categoría de los jugadores (entre 15 y 20 descienden cada año a jugar a segunda categoría individual, independiente de la que tenga su equipo en la liga). En algunas ocasiones, un solo bolo, después de más de 2.500 derribados, es suficiente para determinar el corte y decidir quien juega o no el Campeonato o, lo que es más duro, quien desciende de categoría y quien se mantiene para el próximo año. Todas las bolas son de gran importancia.

Los 16 jugadores clasificados para la fase final, intervienen en un primer día en una doble tirada de octavos de final que, sumados sus bolos, determina los ocho que continúen al día siguiente en la fase final. Tras los cortes de cuartos de final (cuatro se quedan fuera) y los de semifinales (dos quedan eliminados) se disputa la gran final y siempre, en todas las competiciones oficiales, sumando o arrastrando (en el argot bolístico) los bolos de todas las fases.

En este contexto y en estos momentos tan exigentes e intensos psicológicamente fue cuando se administró el cuestionario. Dos son los elementos que destacamos en este trabajo. En primer lugar, el momento en el que se administró el cuestionario: únicamente 25 y 30 minutos antes de comenzar la decisiva fase final de la competición. Consideramos que es un momento idóneo para evaluar el estado de ánimo en un momento muy cercano a competir con la consecuente influencia del estrés precompetitivo en el estado de ánimo del jugador. En segundo lugar la facilidad para evaluar la concordancia entre rendimiento y resultado. En los bolos la influencia entre rendimiento y resultado es precisa y fácil de concretar. 


\section{Método}

\section{Participantes}

En el trabajo participaron los 13 jugadores de bolos de $1^{\text {a }} \mathrm{Ca}-$ tegoría finalistas del LXXIV Campeonato Regional de bolos celebrado en Torrelavega los días 8 y 9 de Agosto de 2014.
Las edades están entre los 21 y 39. No son jugadores profesionales, pues no se dedican exclusivamente a jugar a los bolos. Participan en concursos y torneos en los que ganan premios en metálico y todos tienen firmadas fichas con sus respetivas "peñas" (equipos) por las que perciben una compensación económica. Pertenecen a nueve equipos diferentes de la máxima competición de la modalidad de bolo-palma.

Tabla 1. Situación de los jugadores en el momento de realizar el trabajo. Puesto final en el campeonato regional 2014. Edades. Participaciones en campeonatos regionales anteriores. Títulos conseguidos, subcampeonatos y registros de bolos obtenidos en el campeonato 2014

\begin{tabular}{|c|c|c|c|c|c|}
\hline $\begin{array}{l}\text { PUESTO EN EL CAMPEONATO } \\
\text { REGIONAL }\end{array}$ & EDAD & $\begin{array}{l}\text { PARTICIPACIONES CAMPEONATO } \\
\text { REGIONAL } \\
\end{array}$ & $\begin{array}{c}\mathrm{N}^{\mathrm{o}} \\
\text { TITULOS }\end{array}$ & $\begin{array}{c}\mathrm{N}^{\mathrm{o}} \\
\text { SUBCAMPEONATOS } \\
\end{array}$ & $\begin{array}{c}\text { BOLOS } \\
\text { TOTALES } \\
\end{array}$ \\
\hline $1^{\circ}$ & 36 & 14 & 4 & 6 & $737^{*}$ \\
\hline $2^{o *}$ & 36 & 15 & 1 & - & 694 \\
\hline $3^{\circ}$ & 39 & 21 & 3 & 6 & 522 \\
\hline $4^{o *}$ & 32 & 4 & - & - & 510 \\
\hline $5^{\circ *}$ & 39 & 21 & 7 & 4 & 377 \\
\hline $6^{\circ *}$ & 32 & 0 & - & - & 376 \\
\hline $7^{o}$ & 21 & 2 & - & - & 375 \\
\hline $8^{\circ}$ & 32 & 1 & - & - & 357 \\
\hline $9^{\circ *}$ & 34 & 6 & - & - & 243 \\
\hline $10^{\circ}$ & 42 & 7 & - & - & 242 \\
\hline $11^{\circ}$ & 34 & 2 & - & - & 240 \\
\hline $12^{\circ *}$ & 33 & 2 & - & - & 239 \\
\hline $13^{\circ *}$ & 22 & 0 & - & - & 228 \\
\hline
\end{tabular}

\section{Material}

Se ha utilizado el Profile of Mood States (POMS), McNair, Lorr y Droppleman, 1971) en su versión de 29 ítems, adaptada y validada al español (Fuentes, García-Merita y Balaguer, 1995). La forma abreviada de 29 ítems explica aproximadamente el 94\% de la varianza total de la adaptación del POMS (51 adjetivos). En esta versión los deportistas informan sobre su propio estado de ánimo en relación a cada uno de los ítems del instrumento. El POMS es uno de los instrumentos más utilizados en psicología del deporte para evaluar la percepción de los estados de ánimo precompetitivos. La escala informa sobre cinco estados de ánimo: tensión, cólera, vigor, fatiga, y depresión. La escala presenta una alta consistencia y fiabilidad de medida. (Alphas de: tensión=.83, cólera $=.85$, vigor $=.83$, fatiga $=.82$ y depresión $=.78$ )

\section{Procedimiento}

Los cuestionarios fueron cumplimentados, mediante el empleo de la instrucción: "ahora mismo", entre 30 y 35 minutos antes de comenzar los concursos correspondientes a los octa- vos de final del Campeonato Regional de Bolos. Algunos jugadores ya habían comenzado su participación en el concurso cuando formalizaron el cuestionario. Tras las debidas aclaraciones y objetivo del estudio, los jugadores se mostraron colaboradores y procedieron a cumplimentar voluntariamente el cuestionario. Se aplicó en zonas anejas a la bolera "Severino Prieto" de Torrelavega y fue el propio jugador el que contestó a las cuestiones en presencia de uno de los autores del trabajo, que contestó a las dudas que surgieron en algunos jugadores La duración aproximada para formalizar el cuestionario fue de unos 7-9 minutos.

Las condiciones necesarias para que los jugadores cumplimentaran el instrumento fueron garantizadas en todo el proceso.

\section{Análisis de datos}

Se presentan los bolos conseguidos por todos los jugadores finalistas, así como sus historiales deportivos. Se han analizado y descrito los perfiles de los estados de ánimos precompetitivos de los trece jugadores en orden a la clasificación obtenida. Se han realizado las gráficas de los perfiles del estado de 
ánimo precompetitivo de los jugadores. Se ha elaborado un estudio correlacional entre los factores del POMS, así como contrastes en función de $\mathrm{t}$ de Student y se han comentado las diferencias entre los jugadores según la edad, si habían sido campeones o no y si habían jugado o no un concurso previo al campeonato Regional.

\section{Resultados}

Tabla 2. Valores en los factores del POMS obtenidos por los 13 jugadores finalistas.

\begin{tabular}{lccccc}
\hline CLASIFICACIÓN & TENSIÓN & COLERA & VIGOR & FATIGA & DEPRESIÓN \\
\hline $1^{\circ}$ & 5 & 0 & 24 & 1 & 0 \\
$2^{\circ}$ & 7 & 0 & 15 & 1 & 0 \\
$3^{\circ}$ & 4 & 0 & 11 & 5 & 0 \\
$4^{\circ}$ & 8 & 11 & 8 & 12 & 2 \\
$5^{\circ}$ & 10 & 0 & 12 & 7 & 0 \\
$6^{\circ}$ & 14 & 4 & 11 & 6 & 3 \\
$7^{\circ}$ & 6 & 0 & 22 & 2 & 0 \\
$8^{\circ}$ & 10 & 3 & 14 & 20 & 4 \\
\hline
\end{tabular}

\begin{tabular}{lccccc}
\hline CLASIFICACIÓN & TENSIÓN & COLERA & VIGOR & FATIGA & DEPRESIÓN \\
\hline $9^{\circ}$ & 5 & 18 & 19 & 1 & 2 \\
$10^{\circ}$ & 13 & 6 & 18 & 2 & 0 \\
$11^{\circ}$ & 14 & 7 & 10 & 10 & 5 \\
$12^{\circ}$ & 5 & 11 & 3 & 11 & 8 \\
$13^{\circ}$ & 9 & 0 & 15 & 0 & 1 \\
\hline
\end{tabular}

La figura 1 nos muestra los perfiles de los trece jugadores finalistas ordenados según la clasificación obtenida. Como puede observarse los tres jugadores que hicieron podio presentan un perfil del estado de ánimo precompetitivo idóneo, en consonancia con el perfil "iceberg" en el que el factor vigor se encuentra en niveles superiores al resto de factores. La diferencia entre los perfiles del campeón y del subcampeón y el tercer clasificado se encuentra en una mayor puntuación en el factor fatiga.

El resto de los perfiles, exceptuando al jugador clasificado en la séptima posición presentan perfiles de estados de ánimo precompetitivo muy distorsionados, inadecuados e inconsistentes para afrontar la competición. Ninguno de ellos están en consonancia con el considerado perfil idóneo,"iceberg"

Figura 1. Perfiles de los estados de ánimo precompetitivos de los trece jugadores finalistas del Campeonato Regional de Bolos. consonancia con el perfil "iceberg".

El perfil muestra un adecuado estado de afrontamiento de la competición. En el que el factor vigor se encuentra en niveles óptimos y los demás factores perfectamente nivelados para el correcto rendimiento.

Perfil de estado competitivo previo adecuado para el afrontamiento de la competición. Perfil "iceberg" muy similar al anterior, con el nivel del factor vigor ligeramente inferior y el nivel de factor tensión también ligeramente superior.

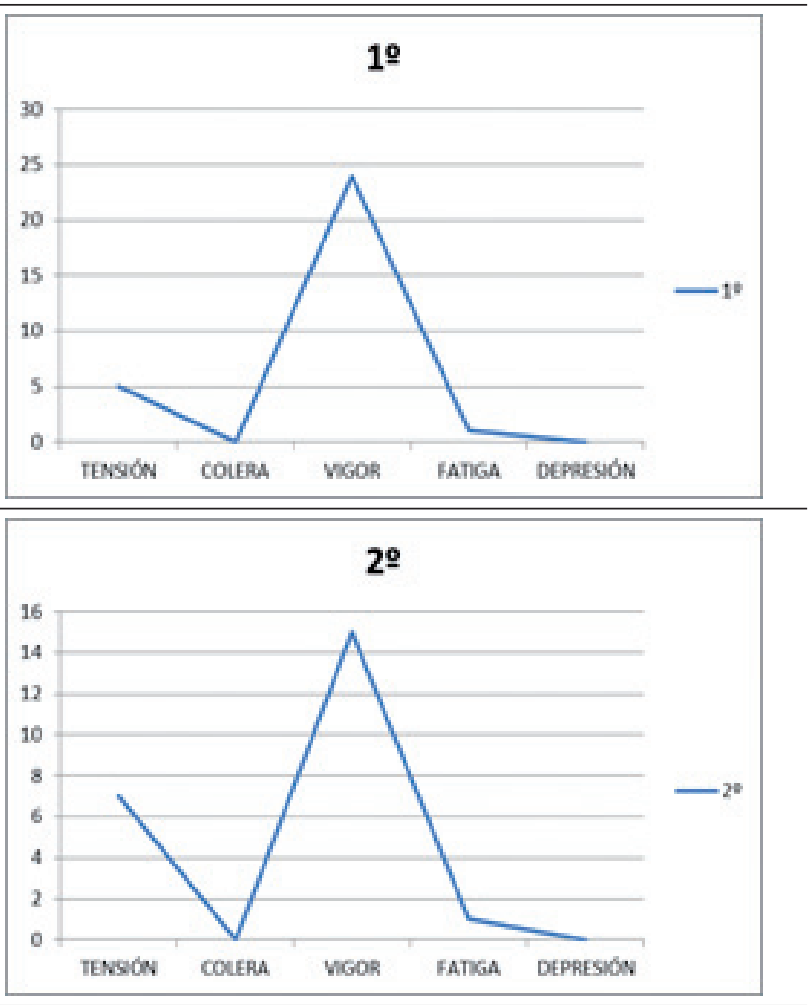


El perfil presenta un nivel de afrontamiento adecuado en la competición con un ligero matiz diferenciador en el factor fatiga, considerado como un factor negativo para el rendimiento.

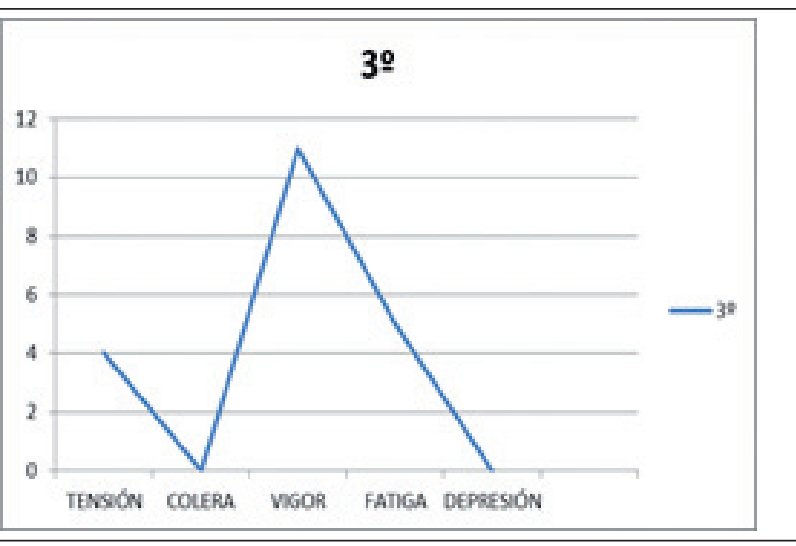

Presenta un inadecuado perfil del estado de ánimo inmediato a la competición.

Este perfil es muy inconsistente con el perfil considerado idóneo y que está establecido como perfecto en los estados previos. Los factores de fatiga y cólera superan al factor vigor. Ambos factores son considerados negativos en la consecución de rendimientos deportivos.

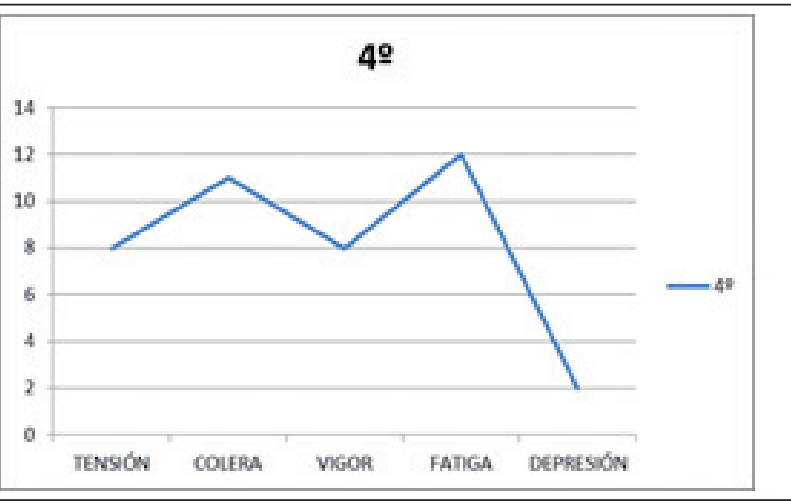

El perfil muestra niveles de los factores tensión y fatiga previas a la competición inadecuadas. Ambos factores se consideran negativos para el rendimiento óptimo.

5은

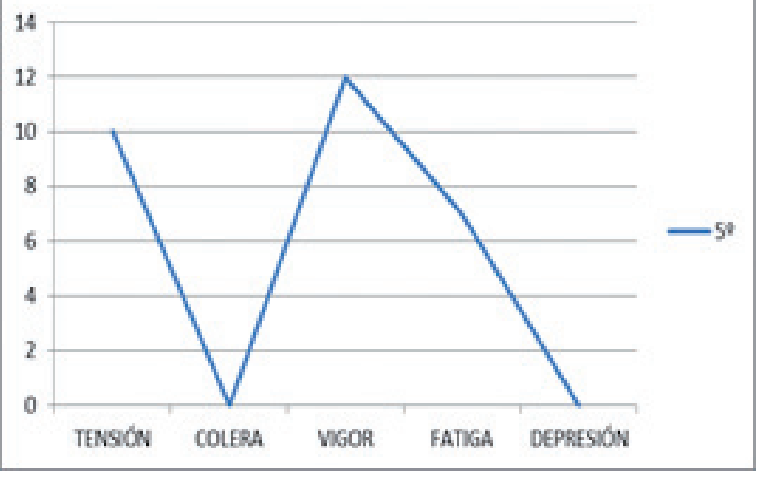

El perfil muestra un estado precompetitivo previo con un factor percibido de tensión por encima del nivel del factor vigor, lo que impide conseguir un perfil "iceberg" característico y apropiado para el buen rendimiento deportivo.

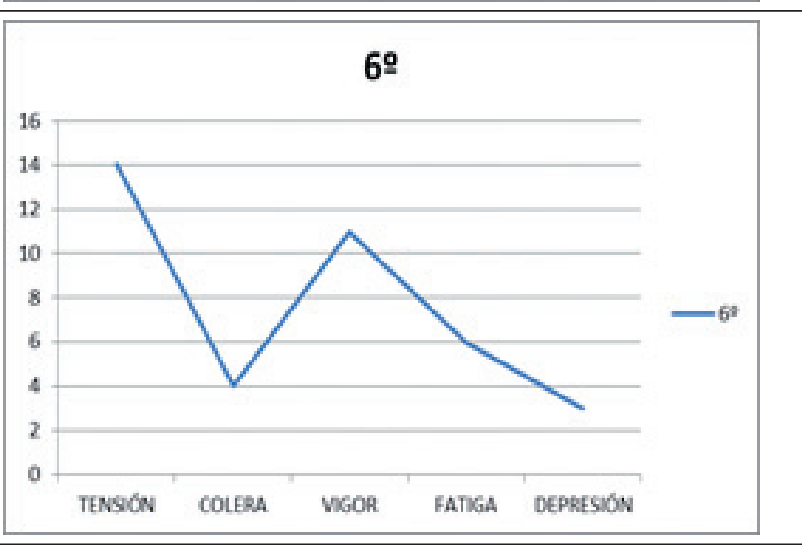


Presenta un buen perfil de estado de ánimo precompetitivo en consonancia con el perfil "iceberg".

El perfil muestra un adecuado estado de afrontamiento de la competición, en el que el factor vigor se encuentra en niveles casi óptimos y los demás factores perfectamente nivelados para el correcto rendimiento.

Perfil de estados de ánimo precompetitivos en el que destaca un nivel elevado del factor fatiga que se encuentra por encima del resto de los factores.

El perfil indica un inadecuado estado anímico para afrontar la competición.

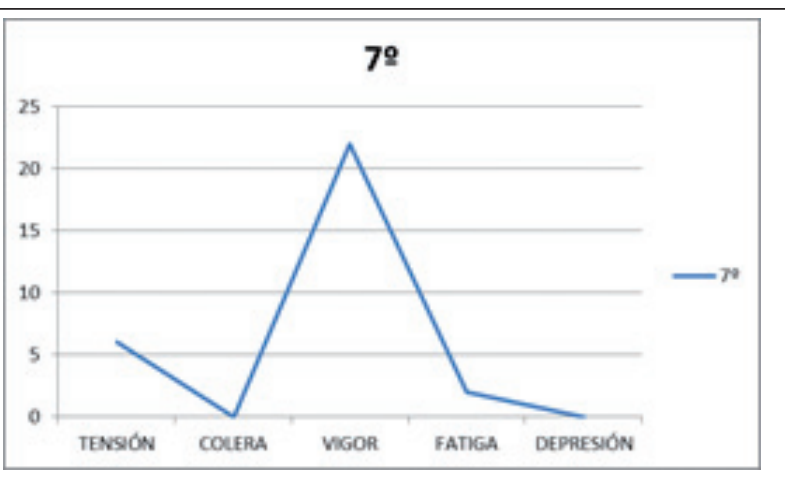

El característico perfil "iceberg” de estados de ánimo considerado el idóneo para competir queda desdibujado al presentar un elevado nivel del factor cólera considerado como un factor negativo para el óptimo rendimiento deportivo.

Presenta un perfil adecuado del estado de ánimo precompetitivo en cuatro de los cinco factores. Si bien la alta puntuación obtenida en el factor tensión distorsiona su perfil de estado anímico. La tensión precompetitiva en un nivel elevado se considera poco apropiada para un deporte de precisión.
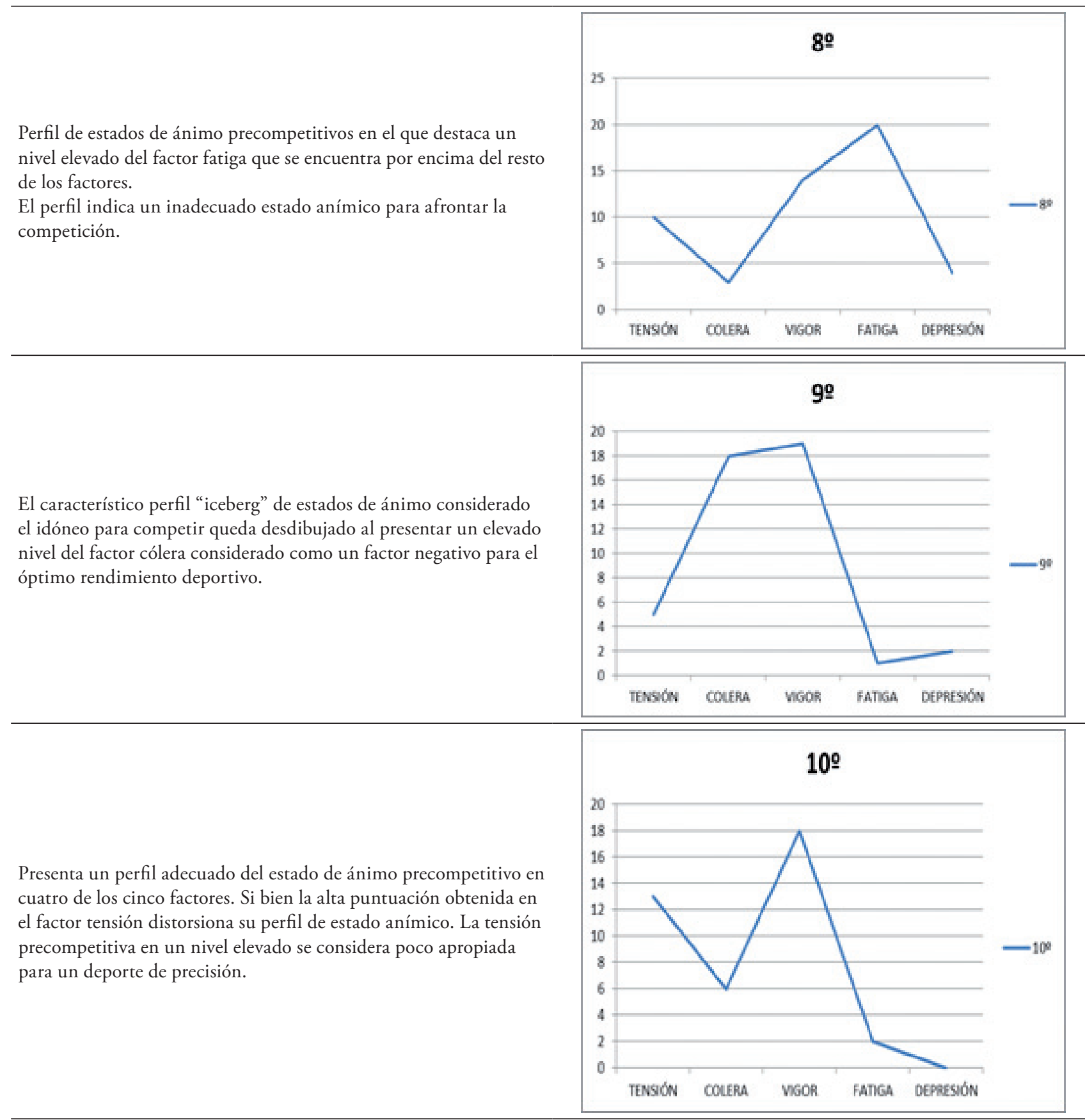
Presenta un perfil del estado de ánimo previo a la competición muy distorsionado. El factor tensión supera al factor vigor que iguala en puntuación factor fatiga. Es un perfil muy inadecuado para la obtención de rendimientos óptimos. Muy alejado del conocido perfil "iceberg".

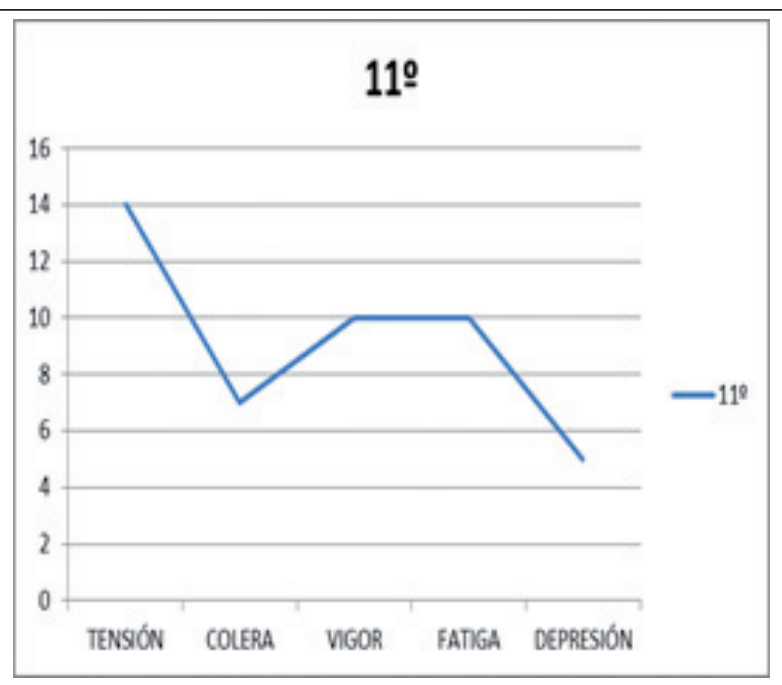

Perfil del estado de ánimo previo a la competición incompatible con el considerado como idóneo para el buen rendimiento deportivo. El nivel del factor vigor en el vértice inferior y por debajo del nivel del resto de los factores.

El segundo jugador más joven de todos los finalistas. Presenta un perfil adecuado del estado de ánimo precompetitivo en cuatro de los cinco factores. Si bien la alta puntuación obtenida en el factor tensión distorsiona su perfil de estado anímico. La tensión precompetitiva en un nivel elevado se considera poco apropiada para un deporte de precisión como los bolos.
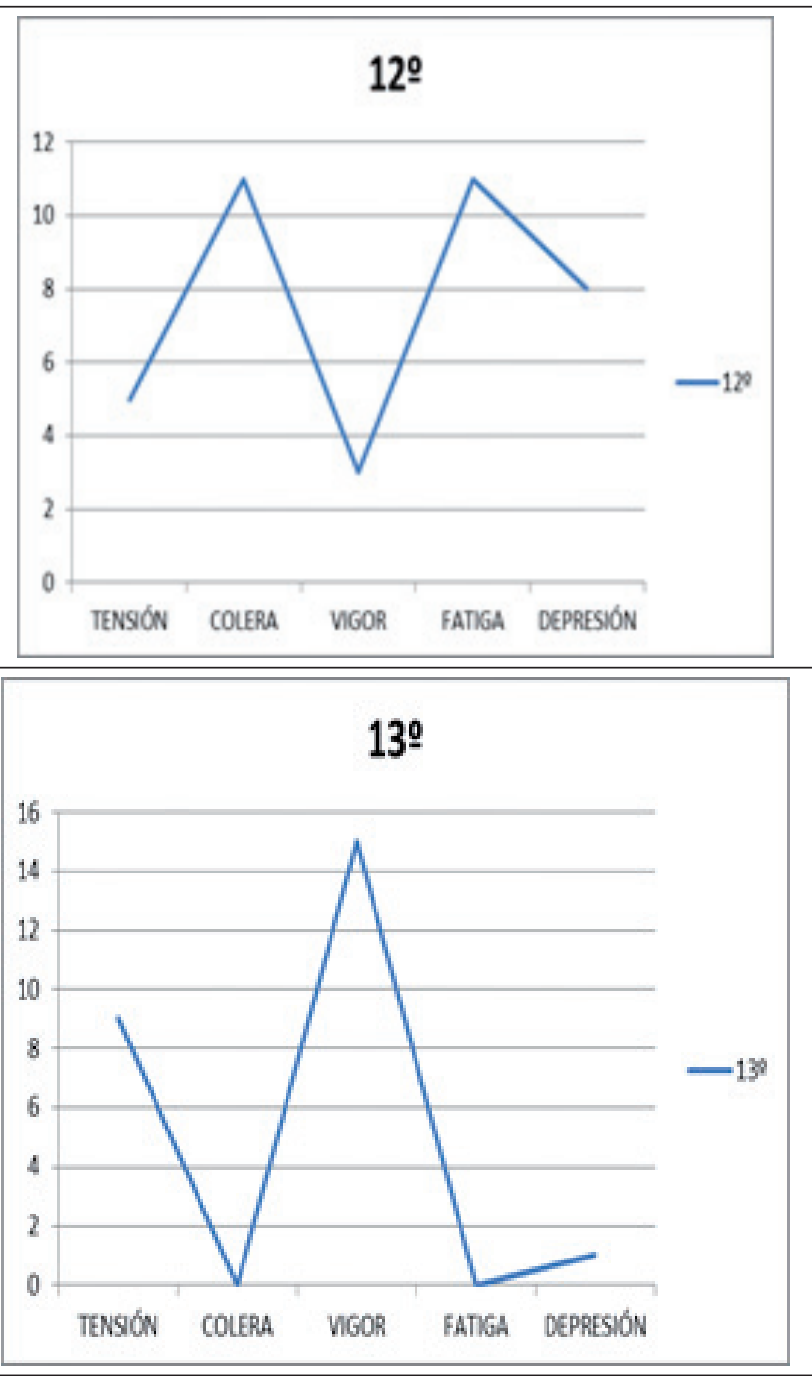


\section{Estudio correlacional}

La tabla 3 recoge las correlaciones entre los cinco factores estudiados en el Profile of Mood States (POMS) y entre cada uno de ellos con el número de bolos derribados por los ju- gadores. Al tratarse de variables continuas, incluye los valores del coeficiente de correlación de Pearson. Se ha ańadido también el nivel de significación estadística de la correlación (bilateral).

Tabla 3. Correlación de Pearson y nivel de significación entre las variables del POMS y los bolos tirados.

\begin{tabular}{|c|c|c|c|c|c|c|}
\hline & & Cólera & Vigor & Fatiga & Depresión & Bolos tirados \\
\hline \multirow[t]{2}{*}{ Tensión } & C. de Pearson &,- 05 &,- 17 &, 24 &, 15 &,- 34 \\
\hline & Sig. (bilateral) &, 87 &, 58 & ,43 & ,64 &, 25 \\
\hline \multirow[t]{2}{*}{ Cólera } & C. de Pearson & &,- 28 & ,19 &, 52 &,- 39 \\
\hline & Sig. (bilateral) & &, 35 &, 54 &, 07 &, 19 \\
\hline \multirow[t]{2}{*}{ Vigor } & C. de Pearson & & &,$- 59\left(^{*}\right)$ &,$- 68\left(^{*}\right)$ & ,43 \\
\hline & Sig. (bilateral) & & &, 04 &, 01 &, 14 \\
\hline \multirow[t]{2}{*}{ Fatiga } & C. de Pearson & & & &, $63\left(^{*}\right)$ &,- 20 \\
\hline & Sig. (bilateral) & & & &, 02 &, 51 \\
\hline \multirow[t]{2}{*}{ Depresión } & C. de Pearson & & & & &,- 43 \\
\hline & Sig. (bilateral) & & & & &, 15 \\
\hline
\end{tabular}

En la figura 1 hemos descrito los perfiles de estado de ánimo precompetitivo de los trece finalistas y hemos comentado los que se consideran perfiles idóneos y los que se consideran inadecuados para la competición. Teniendo en cuenta el perfil "iceberg", considerado idóneo para afrontar la competición deportiva, hemos establecido tres pronósticos:

En primer lugar que la variable vigor correlacione negativamente con todas las demás, es decir, esperamos que a mayor puntuación en esa variable, haya puntuaciones menores en el resto de variables: tensión, cólera, fatiga y depresión.

En segundo lugar, que el resto de variables correlacionen con valores muy pequeños entre sí.

Y, finalmente, que la correlación entre el número de bolos derribados solo sea positiva con la variable vigor y negativa con todas las demás, o dicho de otro modo, que cuanto más alto sea el factor vigor mostrado mayor número de bolos se tirarán, mientras que cuanto mayor sea el valor en el resto de factores, se tirarán menos bolos.

Los datos de la tabla 3. confirman los tres pronósticos anteriores. Con relación al primer aspecto, la variable vigor correlaciona negativamente con las demás, presentando los coeficientes de correlación más altos de la tabla: -,59 con fatiga y -,68 con depresión, de tal modo que la correlación llega a ser significativa. Además, siguiendo a Pérez Juste (2009), los valores obtenidos suponen una "correlación media, marcada, notable". Con la variable tensión la correlación es negativa, tal como hemos pronosticado, pero con un nivel muy bajo: ,- 17 .
Con respecto a la correlación entre el resto de variables, todas presentan valores muy bajos, llegando prácticamente a 0 en el caso de tensión y cólera con un valor de,- 05 . No obstante la excepción es la variable depresión que presenta valores considerables de correlación con cólera ,52 y fatiga, 63, llegando a ser significativa.

El tercer pronóstico que establecimos se confirma claramente en la tabla 3. El número de bolos tirados solo tiene correlación positiva con respecto a la variable vigor, 43, correlacionando negativamente con todas las demás, con valores considerables que van de -,20 con fatiga a -,42 con depresión.

\section{Diferencias entre los perfiles}

Hemos estudiado las diferencias entre los perfiles del POMS en función de la edad de los jugadores, el que hayan sido campeones o no con anterioridad a 2014 y que hubieran jugado o no un concurso de bolos previo al propio Campeonato Regional.

Las tablas 4 y 5 recogen los rangos en los cinco factores del POMS y en la variable bolos tirados, tras dividir la muestra en dos grupos en función de tener más o menos de 35 años.

Observamos, en primer lugar, que los mayores de 35 años tienen menos tensión, cólera, fatiga y depresión y, por el contrario presentan mayor puntuación en vigor. Por lo tanto, los mayores de 35 años consiguen resultados más en consonancia con el perfil iceberg que se obtiene tras la aplicación del POMS. 
Tabla 4. Rangos en función de tener más o menos de 35 años.

\begin{tabular}{llccc}
\hline & Edad & N & $\begin{array}{c}\text { Rango } \\
\text { promedio }\end{array}$ & $\begin{array}{c}\text { Suma de } \\
\text { rangos }\end{array}$ \\
\hline Tensión & Menos de 35 años & 8 & 7,88 & 63,00 \\
& Más de 35 años & 5 & 5,60 & 28,00 \\
Cólera & Menos de 35 años & 8 & 7,50 & 60,00 \\
& Más de 35 años & 5 & 6,20 & 31,00 \\
Vigor & Menos de 35 años & 8 & 6,63 & 53,00 \\
& Más de 35 años & 5 & 7,60 & 38,00 \\
Fatiga & Menos de 35 años & 8 & 7,56 & 60,50 \\
\hline
\end{tabular}

\begin{tabular}{llccc}
\hline Edad & N & $\begin{array}{c}\text { Rango } \\
\text { promedio }\end{array}$ & $\begin{array}{c}\text { Suma de } \\
\text { rangos }\end{array}$ \\
\hline \multirow{3}{*}{ Depresión } & Más de 35 años & 5 & 6,10 & 30,50 \\
& Menos de 35 años & 8 & 8,56 & 68,50 \\
& Más de 35 años & 5 & 4,50 & 22,50 \\
& Menos de 35 años & 8 & 5,25 & 42,00 \\
& Más de 35 años & 5 & 9,80 & 49,00 \\
\hline
\end{tabular}

En segundo lugar, vemos que las diferencias son estadísticamente significativas en depresión y en que tiran más bolos los jugadores de más de 35 años.

Tabla 5. Estadísticos de contraste en función de tener más o menos de 35 años.

\begin{tabular}{lcccccc}
\hline & Tensión & Cólera & Vigor & Fatiga & Depresión & Bolos tirados \\
\hline U de Mann-Whitney & 13,000 & 16,000 & 17,000 & 15,500 & 7,500 & 6,000 \\
W de Wilcoxon & 28,000 & 31,000 & 53,000 & 30,500 & 22,500 & 42,000 \\
Sig. asintót. (bilateral) &, 301 &, 537 &, 660 &, 507 &, 054 &, 039 \\
\hline
\end{tabular}

Respecto a posibles diferencias de estado ánimo precompetitivo entre los jugadores más experimentados, es decir; aquellos que habían tenido más participaciones en campeonatos regionales e incluso haber obtenido algún título, analizamos si había diferencias en los factores del POMS y en la variable bolos tirados, en función de haber ganado campeonatos regionales de bolos en ediciones anteriores.

En la tabla 6 se muestra que aquellos jugadores que han sido campeones regionales en alguna ocasión presentan menores puntuaciones en los factores de: tensión, cólera, fatiga y depresión que aquellos que no han sido nunca campeones. Estos datos se corresponden con el perfil idóneo de estados de ánimo precompetitivos a través del POMS. Sin embargo ambos grupos presentan el mismo nivel de vigor, cuando lo previsible sería que los que han sido campeones presentaran un rango superior al de no campeones.

Por otro lado, no hemos encontrado que esas diferencias sean significativas, excepto en que los que han sido campeones han tirado más bolos en este campeonato.
Tabla 6. Rangos en función de haber sido o no campeones. Experiencia competitiva.

\begin{tabular}{llccc}
\hline & Campeones & N & $\begin{array}{c}\text { Rango } \\
\text { promedio }\end{array}$ & $\begin{array}{c}\text { Suma de } \\
\text { rangos }\end{array}$ \\
\hline Tensión & Campeones & 4 & 4,25 & 17,00 \\
& No campeones & 9 & 8,22 & 74,00 \\
Cólera & Campeones & 4 & 5,50 & 22,00 \\
& No campeones & 9 & 7,67 & 69,00 \\
Vigor & Campeones & 4 & 7,00 & 28,00 \\
& No campeones & 9 & 7,00 & 63,00 \\
Fatiga & Campeones & 4 & 6,25 & 25,00 \\
& No campeones & 9 & 7,33 & 66,00 \\
Depresión & Campeones & 4 & 4,75 & 19,00 \\
& No campeones & 9 & 8,00 & 72,00 \\
Bolos tirados & Campeones & 4 & 11,25 & 45,00 \\
& No campeones & 9 & 5,11 & 46,00 \\
\hline
\end{tabular}

Tabla 7. Estadísticos de contraste en función de haber sido o no campeones. Experiencia competitiva.

\begin{tabular}{lcccccc} 
& Tensión & Cólera & Vigor & Fatiga & Depresión & Bolos tirados \\
\hline U de Mann-Whitney & 7,000 & 12,000 & 18,000 & 15,000 & 9,000 & 1,000 \\
\hline W de Wilcoxon & 17,000 & 22,000 & 63,000 & 25,000 & 19,000 & 46,000 \\
Sig. asintót. (bilateral) &, 087 &, 329 & 1,000 &, 641 &, 143 &, 008 \\
\hline
\end{tabular}

Con relación a las diferencias en función de haber jugado o no un concurso previo al Campeonato Regional, los resultados se recogen en las tablas 8 y 9 .

En los contrastes estadísticos no han aparecido diferen- cias significativas en ninguno de ellos. Los que jugaron un concurso previo presentan mayor rango promedio en cólera y menor en todos los demás: tensión, fatiga, depresión y vigor. 
Tabla 8. Rangos en función de haber jugado o no un concurso previo.

\begin{tabular}{lllcc}
\hline & Concurso previo & N & Rango promedio & Suma de rangos \\
\hline Tensión & Sí jugaron concurso previo & 7 & 5,36 & 37,50 \\
Cólera & No jugaron concurso previo & 6 & 8,92 & 53,50 \\
& Sí jugaron concurso previo & 7 & 7,14 & 50,00 \\
Vigor & No jugaron concurso previo & 6 & 6,83 & 41,00 \\
& Sí jugaron concurso previo & 7 & 5,93 & 41,50 \\
Fatiga & No jugaron concurso previo & 6 & 8,25 & 49,50 \\
& Sí jugaron concurso previo & 7 & 6,57 & 46,00 \\
Depresión & No jugaron concurso previo & 6 & 7,50 & 45,00 \\
& Sí jugaron concurso previo & 7 & 6,79 & 47,50 \\
Bolos tirados & No jugaron concurso previo & 6 & 7,25 & 43,50 \\
& Sí jugaron concurso previo & 7 & 7,00 & 49,00
\end{tabular}

Tabla 9. Estadísticos de contraste en función de haber jugado o no un concurso previo.

\begin{tabular}{lcccccc}
\hline & Tensión & Cólera & Vigor & Fatiga & Depresión & Bolos tirados \\
\hline U de Mann-Whitney & 9,500 & 20,000 & 13,500 & 18,000 & 19,500 & 21,000 \\
W de Wilcoxon & 37,500 & 41,000 & 41,500 & 46,000 & 47,500 & 42,000 \\
Sig. asintót. (bilateral) &, 098 &, 880 &, 283 &, 666 &, 821 & 1,000 \\
\hline
\end{tabular}

\section{Discusión}

Los datos obtenidos en el presente trabajo muestran que los jugadores finalistas en el Campeonato Regional de bolos se enfrentan a la competición con diferentes estados psicológicos previos, en este caso los estados de ánimo analizados mediante el POMS.

Los perfiles muestran que los jugadores que ocuparon los tres primeros puestos del Campeonato Regional presentan el típico perfil "iceberg" en los que la escala vigor se sitúa por encima del resto de las variables. Coincidiendo con los trabajos de Beedie, Terry y Lane, (2000), ante jugadores con el mismo nivel de experiencia y habilidad, los resultados en la competición se explican en función de la adecuación o no del perfil iceberg. Destaca el factor vigor del campeón (.24) la mejor puntuación de todos los participantes. El vigor es la variable positiva del POMS (Fuentes et al., 1995). La obtención de este perfil predispone a los jugadores a obtener rendimientos óptimos (Morgan, 1980; O`Connor, 2006). En nuestro estudio este rendimiento óptimo es ratificado por la obtención de resultados muy altos del campeón. Creemos que es importante destacar que el campeón presentó el mejor resultado, según el POMS, del estado previo a la competición, en la que consiguió el record absoluto de bolos derribados en toda la historia de los campeonatos regionales. En la figura 1 se pueden comparar las gráficas de los perfiles de los ju- gadores clasificados en los tres primeros puestos y las de los jugadores clasificados en los tres últimos puestos.

En la tabla 6 se recogen los resultados en función de la experiencia competitiva, es decir, haber jugado finales del Campeonato Regional. Hemos encontrado que el valor en el factor vigor obtiene el mismo rango en los dos grupos. Los jugadores con menor experiencia son los clasificados en los puestos del $5^{\circ}$ al $13^{\circ}$ del Campeonato Regional, todos ellos, con excepción del jugador que ocupó el $7^{\circ}$ puesto, presentaban perfiles en el POMS distorsionados y poco apropiados para conseguir rendimientos y resultados óptimos. Los resultados de este trabajo son similares a otros (Terry, 1995; Rowley, Landers, Kyllo y Etnier, 1995) que inciden en que deportistas de distintos niveles muestran similares puntuaciones del estado de ánimo en el factor vigor. Por lo tanto, ambos grupos: experimentados, aquellos que han participado en más campeonatos regionales y que han sido campeones regionales, y no experimentados, que nunca han sido campeones regionales, presentan el mismo nivel en el factor vigor. Sin embargo, el grupo de experimentados obtiene un perfil más cercano al perfil iceberg en el resto de los factores. Se puede visualizar este dato comparando en la figura 1 los perfiles de los jugadores que han conseguido los cuatro primeros puestos y el resto.

Los jugadores mayores de 35 ańos consiguen puntuaciones menores en los factores negativos tensión, cólera, fatiga y 
depresión y, por el contrario presentan mayor puntuación en vigor. Por lo tanto, los jugadores finalistas del Campeonato Regional de bolos mayores de 35 años obtienen resultados más en consonancia con el perfil iceberg que se obtiene tras la aplicación del POMS. Estos resultados parecen vislumbrar, como ya planteaban Balaguer, Fuentes, Meliá, García-Merita, y Pérez Recio (1993), que la intensidad o cantidad de práctica deportiva permite cambios positivos en los estados de ánimo precompetitivos.

En nuestro estudio los jugadores más experimentados, es decir, que han participado en más campeonatos y de más edad, son también, los que han sido más veces campeones regionales, lo que presupone una mayor calidad técnico táctica, son los que presentan perfiles de estado de ánimo precompetivos más idóneos. Estos resultados están en concordancia con los obtenidos por De la Vega, R., Ruíz, R., García, G., del Valle, S. (2011) con jugadores de fútbol en los que los jugadores titulares, teóricamente los considerados mejores, tienen un mejor perfil de los estados de ánimo antes de enfrentarse a un partido que los suplentes.

Los jugadores que cumplimentaron su cuestionario una vez que había jugado uno de los concursos de la final, y por tanto venían con un resultado previo inmediato, presentaban un rango mayor en cólera que el resto de los jugadores y menor en todos los demás factores del estado de ánimo independientemente de los resultados obtenidos. La condición de haber jugado previamente, en principio, podría interpretarse como una forma de alteración del estado de ánimo tanto en forma positiva como negativa, según los resultados obtenidos. El modelo de zona individual de funcionamiento óptimo (IZOF, en sus siglas en inglés) propuesto por Hanin (1999) que pretende explicar la influencia de la experiencia individual sobre el rendimiento deportivo, podría explicar esta situación. Es decir, que los estados de ánimo previos a una competición dependen de la interpretación individualizada que realiza el deportista. Además, como refieren los trabajos de Andrade, E., Arce, C. y Seoane, G. (2000) hay otros factores que influyen en el rendimiento deportivo y no sólo los psicológicos, factores como el esfuerzo, la presión competitiva y los estresores de la vida laboral, familiar etc. pueden influir de forma diferente en el rendimiento. En el caso de la competición de bolos un rango en el factor cólera superior a los otros rasgos supondría un jugador incapaz de concentrase, confundido y desorientado, sin embargo tres de estos jugadores terminaron entre los cuatro primeros del campeonato.

\section{Conclusiones}

El POMS se ha utilizado por primera vez en la modalidad de bolos. El empleo del POMS para el estudio de los estados de ánimo previos a la competición en la modalidad de bolos ha demostrado ser también una herramienta útil para predecir el rendimiento deportivo y los resultados. Los jugadores con perfiles de estados de ánimo más idóneos según el POMS son los que han conseguido los mejores resultados.

El POMS ha aportado información y resultados similares en esta modalidad al encontrado en otras modalidades deportivas. Los jugadores de más edad y con mayor experiencia en niveles de competición muy exigentes en el deporte de los bolos, presentan los perfiles de estado de ánimo previos más adecuados para rendimientos óptimos y se adecuan más al nivel de "perfil iceberg". Estos resultados nos permiten reflexionar sobre la relación entre estados de ánimo precompetitivos y experiencia y nivel competitivo y edad. En futuros trabajos en esta modalidad deportiva y modalidades similares se deberá profundizar sobre formas de entrenamiento mental para ayudar a los jugadores más jóvenes e inexpertos a crear las condiciones anímicas más apropiadas para enfrentarse a competiciones transcendentes y exigentes.

Otra futura línea de futuros estudios debería abordar la mayor o menor consecuencia que tienen los estados de ánimo previos a la competición sobre la mejor técnica y experiencia de los jugadores para conseguir mejores resultados. En este sentido, los jugadores menos experimentados y más jóvenes, quizás, podrían igualar más sus rendimientos y resultados a los más experimentados, recibiendo el entrenamiento psicológico que les permitiera preparar apropiadamente su estado de ánimo para enfrentarse a la competición en un contexto de máxima exigencia como son las finales de un Campeonato Regional como el que hemos comentado en este trabajo.

\section{Bibliografia}

1. Andrade, E.M, Arce, C., y Seoane, G. (2000). Aportaciones del POMS a la medida del estado de ánimo de los deportistas: estado de la cuestión, Revista de Psicología del Deporte. 9(1/2) 7-20.

2. Arruza, J., Balagué, G. y Arrieta, M. (1998). Rendimiento deportivo e influencia del estado de ánimo, de la dificultad percibida y de la autoeficacia en el deporte de alta competición. Revista de Psicología del Deporte. 7(2) 193-204.

3. Balaguer, I., Fuentes, I., Melia, J.L., García-Merita, M.L., Pérez Recio, G. (1993) El perfil de los estados de ánimo (POMS): baremos para estudiantes valencianos y su aplicación en el contexto deportivo. Revista de Psicología del deporte. 4, 39-52.
4. Beedie, C.J., Terry, P.C. y Lane, A. (2000). The Profile of Mood States and athletic performance: two meta-analyses. Journal of Applied Sport Psychology. 12(1).49-68.

5. Bonete, E., Moya, M. y Suay, F. (2009). La subescala confusión del POMS como indicador del impacto de la carga de entrenamiento en corredores de fondo y medio fondo. Revista Iberoamericana de Psicología del Ejercicio Físico y del Deporte, 4(2).

6. De la Vega, R., Ruiz. R., García-Mas. A., Balagué, G., Olmedilla, A. y del Valle, S. (2008). Consistencia y fluctuación de los estados de ánimo en un equipo de fútbol profesional durante una competición de playoff. Revista de Psicología del deporte, 17(2) 241-251. 
7. De la Vega, R., Ruiz, R., García G. y Del Valle, S. (2011). El estado de ánimo precompetitivo en un equipo de fútbol profesional: un estudio entre jugadores titulares y suplentes. Cuadernos de Psicología del Depor te. 11(2) 107-117.

8. De la Vega, R., Galán, A., Ruiz, A.; y Tejero, C. (2013).Estado de ánimo precompetitivo y rendimiento percibido en Boccia Paralímpica. Revista de Psicología del Deporte. 22(1) 39-45.

9. Díaz, J. (2010) El entrenamiento mental de los jugadores de bolos. Preparación para el alto rendimiento. Cantabria. Federación Cántabra de Bolos.

10. Fuentes, I., Balaguer, I., Meliá, J.J., y García-Merita, M.L. (1995) Forma abreviada del Perfil de los Estados de Ánimo (POMS). Actas del V Congreso Nacional de Psicología de la Actividad Física y el Deporte.29-39 Valencia. Espańa.

11. González, J. G. (2007). Herramientas aplicadas al desarrollo de la concentración en el alto rendimiento deportivo. Cuadernos de psicología del deporte, $7(1), 61-70$.

12. Hanin, Y, (1999) Emotions in Sport. England: Human Kinetis

13. Hassmen, H. y Blomstrand, E. (1995). Mood state relationships and soccer team performance. The Sport Psychologist, 2917-308.

14. Hernández, R., Torres-Luque, G. y Olmedilla, A. (2009) Relations among training volume, body weight and profile of moods states for elite judoka during a competitive period. Perceptual and Motor Skills, 109(3), 128-133.

15. Lazarus, R.S. (2000). How emotions influence performance in competitive sport. The Sport Psychologist. 229 -252.

16. Leunes, A., y Hayward, S.A., (1989) Annotated bibliography on the Profile of Mood States in Sport, 1975-1988. Journal of Sport Behavior, 11,213-240.

17. McNair, D.M., Lorr, M. y Droppleman, L.F.1971). Manual for the Profile of Mood States. San Diego, CA: Educational and Industrial Testing Services.

18. Morgan, W.P. (1980) Test of champions. Psychology Today, Julio.92-99

19. Morgan, W.P., Costill, D.L., Flynn, M.G., Ranglin, J.S. y O`Connor, P.J. (1988). Mood disturbance following increased training in swim- mers. Medicine and science in Sports and exercise, 20, 408-414.

20. Morgan, W.P. y Johnson, R.W. (1977). Psychological characterization of the elite wrestler: a mental health model. Medicine and science in Sports and exercise, 9, 55-56.

21. Morgan, W.P. y Pollock, M.L. (1977) Psychologic characterization of the elite distance runner. Annals of the New York Academy of Science, 301, 382-403.

22. Nagle, F.J., Morgan, W.P., Hellickson, R.O., Serfass, R.C., y Alexander, J.F. (1975).Spotting success traits in Olympic contenders. The Physicians and Sports Medicine, 18 85-92.

23. O`Connor, P.J. (2006). Mental Energy: Assessing the Mood Dimension. Nutrition Revews,64(7) S7-S9.

24. Pérez, G., Solanas, A. y Ferrer, M. (1993). Monitorización continua del estado de ánimo en nadadores. Apunts.30 116, 87-97.

25. Pérez Juste, R. (2009). Estadística aplicada a la educación. Madrid: Pearson.

26. Rowley, A., Landers, D., Kyllo, L. y Etnier, J. (1995) Does the iceberg profile discriminate between successful and less successful athletes?. A meta-análisis. Journal of Sport and exercise Psychology, 17, 185-199.

27. Ruiz, M.C., y Hanin, Y.L.(2004) Athlete`s self-percepcions of optimal states in karate. An application of the IZOF model. Revista de Psicologia del Deporte. 13 (2) 229-244.

28. Sánchez, A., González, E., Ruíz, M., San Juan, M., Abando, J., De Nicolas, L. et al. (2001)Estados de ánimo y rendimiento deportivo en futbol. :Existe la ventaja de Jugar en casa? Revista de Psicología del Deporte.197-210.

29. Segura, J., Molist, E., Arcarons, M.y Piqué, N. (1999) Práctica deportiva y estado de ánimo en deportistas discapacitados físicos. Apunts. Medicina de l'sport, 131,11-18.

30. Terry, P.C. (1995). Discriminat capability of pre-performance mood state profiles during 1993-94 World cup Bobsleigh. Journal of Sport Sciences, 13(1) 77-78

31. Terry, P.C. (1995). Discriminat effectiveness of psychological states measures in predicting performance outcome in field hockey trialists. Perceptual and Motor Skills, 82 371-373. 